\title{
Note on Bivariate Linear Interpolation for Analytic Functions
}

\author{
By Walter Gautschi
}

1. Introduction. As more tables of complex analytic functions are being produced* $^{*}$ the problem of complex interpolation has recently obtained increasing attention. The research has mainly been directed toward formulas of high accuracy based on Lagrangian and Lagrange-Hermitian interpolation (see, e.g., [9], [10], [11] and the literature quoted there). In the present note we are dealing with the simpler means of bivariate interpolation, having an occasional computer in mind with only moderate accuracy requirements. It will be shown that bivariate interpolation enjoys special properties if applied to the real and imaginary part of an analytic function and we shall indicate possibilities for slightly improving the accuracy. Although we are restricting ourselves to linear interpolation, similar arguments could be used for bivariate quadratic interpolation.

2. Bivariate cartesian interpolation. Let $f(z), z=x+i y$, be a single-valued analytic function in a domain $D$ of the complex plane. We consider first bivariate interpolation with respect to a rectangle $R$ in $D$.

By a translation and rotation of the $z$-plane we can fix $R$ to be the rectangle $|x| \leqq \frac{1}{2} h,|y| \leqq \frac{1}{2} k$ for some $h>0, k>0$ with $h \geqq k$. We shall adopt the notations

$$
z_{p, q}=\frac{1}{2} p h+\frac{1}{2} i q k, \quad f_{p, q}=f\left(z_{p, q}\right),
$$

so that points $z_{p, q}$ in the closed rectangle are characterized by $|p| \leqq 1,|q| \leqq 1$. We also set $k=\sigma h(\sigma \leqq 1)$.

Given the values of $f(z)$ at the vertices of $R$, bivariate linear interpolation to evaluate $f_{p, q}$ proceeds as follows. First we interpolate linearly along two parallel sides of $R$, say the sides parallel to the $x$-axis, to obtain

$f_{p,-1}^{*}=\frac{1}{2}(1-p) f_{-1,-1}+\frac{1}{2}(1+p) f_{1,-1}, \quad f_{p, 1}^{*}=\frac{1}{2}(1-p) f_{-1,1}+\frac{1}{2}(1+p) f_{1,1}$.

Then we interpolate linearly in the $y$-direction between these two values:

$$
f_{p, q}^{*}=\frac{1}{2}(1-q) f_{p,-1}^{*}+\frac{1}{2}(1+q) f_{p, 1}^{*} \text {. }
$$

Thus

$$
\begin{aligned}
f_{p, q}^{*}=\frac{1}{4}\left\{(1-p)(1-q) f_{-1,-1}\right. & +(1+p)(1-q) f_{1,-1} \\
& \left.+(1-p)(1+q) f_{-1,1}+(1+p)(1+q) f_{1, !}\right\}
\end{aligned}
$$

In order to investigate the error $f_{p, q}-f_{p, q}^{*}$ we expand this difference into powers

Received June 2, 1958. This paper was prepared under a National Burealu of Standards contract with American University.

* Among the more recent tables we mention [1]-[8]. 
of $h$ by means of Taylor's series. We find

$$
\begin{aligned}
& f\left(z_{p, q}\right)-f_{p, q}^{*}=\frac{h^{2}}{8}\left(p^{2}-\sigma^{2} q^{2}-1+\sigma^{2}\right) f^{\prime \prime}(0) \\
& +\frac{h^{3}}{48}\left\{p^{3}-3 \sigma^{2} p q^{2}+\left(3 \sigma^{2}-1\right) p+i \sigma\left[3 p^{2} q-\sigma^{2} q^{3}-\left(3-\sigma^{2}\right) q\right]\right\} f^{\prime \prime \prime}(0) \\
& \quad+O\left(h^{4}\right) .
\end{aligned}
$$

From (3) we note the following facts:

(i) Bivariate linear interpolation based on the vertices of a rectangle $R$ with side ratio $\sigma$ has an error of order $h^{2}$ except for points $z_{p, q}$ lying on the hyperbolas $p^{2}-\sigma^{2} q^{2}-1+\sigma^{2}=0$ where the error is of order $h^{3}$;

(ii) the interpolation error is largest in absolute value in the neighborhood of the midpoints of the larger sides of $R$;

(iii) in case of a square $(\sigma=1)$ the interpolation error is of order $h^{2}$ except for points along the diagonals, where it is of order $h^{3}$ and the center of the square, where it is of order $h^{4}$.

At the center of a rectangle the result of bivariate linear interpolation is simply given by the arithmetic mean

$$
f_{0,0}^{*}=\frac{1}{4}\left(f_{-1,-1}+f_{1,-1}+f_{-1,1}+f_{1,1}\right) .
$$

The relation (4) in case of a square is often used for the numerical solution of the Laplace equation. In this connection the error property mentioned last in (iii) is a well known fact.

The magnitude of the coefficient of $h^{2} f^{\prime \prime}(0) / 8$ in (3) is shown in Figs. 1 and 2 for the cases of a rectangle $\left(\sigma=\frac{1}{2}\right)$ and a square $(\sigma=1)$.

3. Successive bivariate interpolations. Suppose now that $f(z)$ be tabulated at the nodes of a square grid with grid width $h$. The simple device of forming the

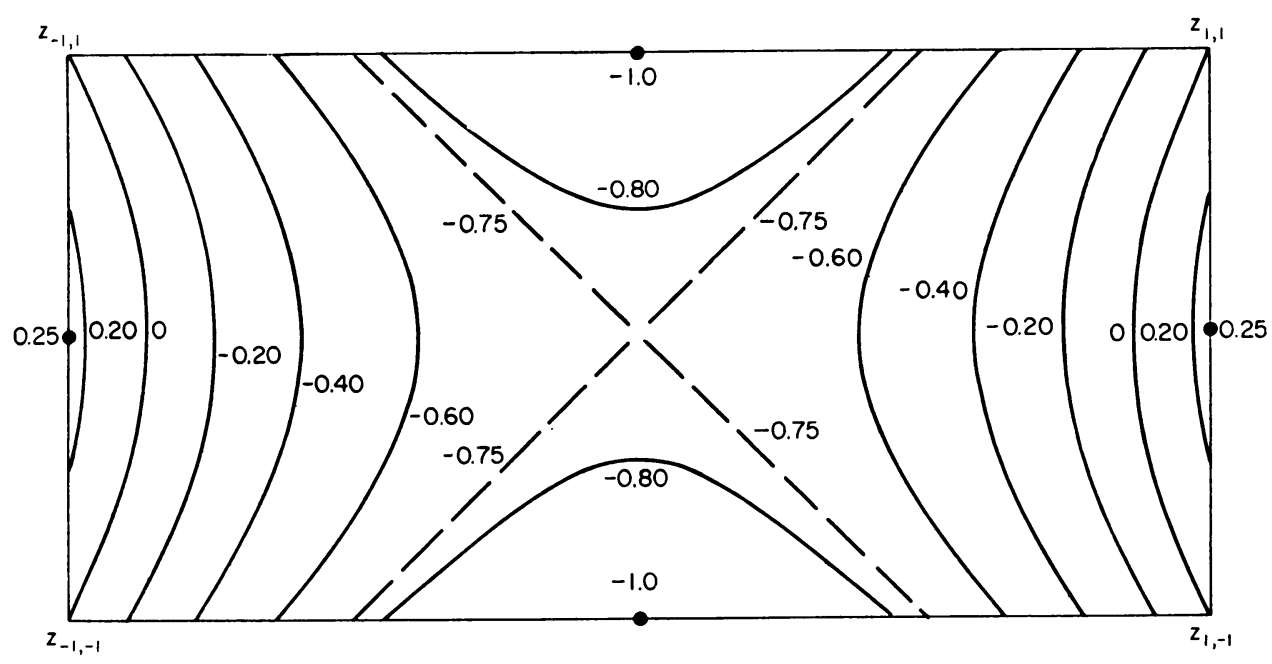

FIG. 1.-Curves of constant interpolation error in a rectangle (neglecting third order terms). 


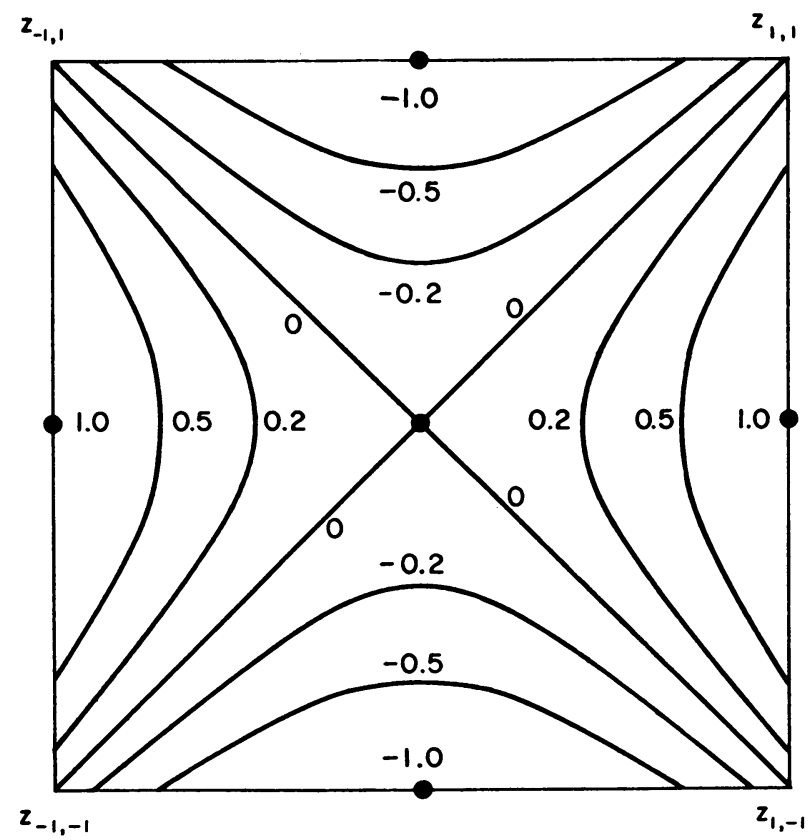
terms).

Fig. 2.-Curves of constant interpolation error in a square (neglecting third order

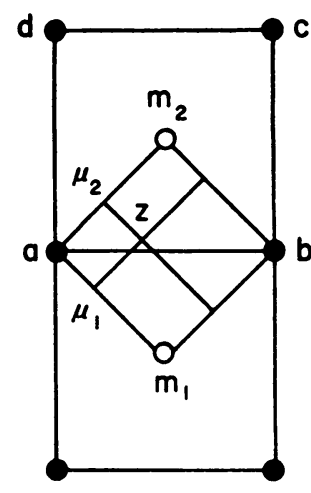

Fig. 3a. Grid line.

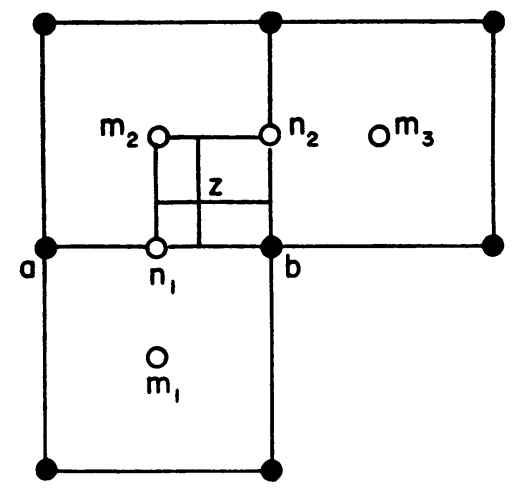

Fig. 3b. Grid line.

arithmetic mean (4) yields the value of $f$ at the center of a grid square accurately to $0\left(h^{4}\right)$. Therefore we can generate, within this accuracy, a new table of $f$ with respect to the "dual" grid consisting of all centers of the original grid. With the union of both grids as a new starting grid the process can be repeated indefinitely. Thus, in principle, it is possible to evaluate $f(z)$ for any $z$ with an error of essentially $0\left(h^{4}\right)$ by a succession of linear bivariate interpolations.

For the purpose of interpolation, of course, one should try to introduce new grid points as economically as possible and in such a way that the given point $z$ will be close to the center of a new grid square.

To illustrate, assume that $z$ lies near the midsection of a grid line $a b$ (see Fig. 3a.). 
In this case we evaluate $f\left(m_{1}\right), f\left(m_{2}\right)$ at the centers $m_{1}$ and $m_{2}$ by averaging and then apply bivariate linear interpolation to the square $a m_{1} b m_{2}$. It should be noted that the parts with respect to this square are

$$
p_{1}{ }^{\prime}=\frac{\left|a-\mu_{1}\right|}{\left|a-m_{1}\right|}=p_{1}-p_{2}, \quad p_{2}{ }^{\prime}=\frac{\left|a-\mu_{2}\right|}{\left|a-m_{2}\right|}=p_{1}+p_{2},
$$

if $p_{1}, p_{2}$ are the corresponding parts with respect to the square $a b c d$ and $p_{1}>p_{2}>0$.

In a case like that shown in Fig. 3b we may first compute $f\left(m_{1}\right), f\left(m_{2}\right), f\left(m_{3}\right)$ by averaging and $f\left(n_{1}\right), f\left(n_{2}\right)$ again by averaging. Then we can use the square $n_{1} b n_{2} m_{2}$ for bivariate interpolation.

4. Bivariate polar interpolation. Let $f(w), w=\rho e^{i \theta}$, be a single-valued analytic function defined in a certain domain $E$ of the $w$-plane. We consider now bivariate interpolation with respect to a sector $S$ of an annulus located in $E$. Without restricting generality we can assume that $S$ is the sector $r \leqq \rho \leqq r+\Delta r,|\theta| \leqq$ $\frac{1}{2} \Delta \theta$. In analogy with (1) we denote

$$
\begin{gathered}
w_{p, q}=\left(r+\frac{p+1}{2} \Delta r\right) \exp \left(\frac{1}{2} i q \Delta \theta\right) \quad(|p| \leqq 1,|q| \leqq 1), \\
f_{p, q}=f\left(w_{p, q}\right),
\end{gathered}
$$

and also set $\Delta \theta=\tau \Delta r$.

With these notations the formula for bivariate polar interpolation is identical with formula (2). Its properties are readily deduced from the results of section 2 by means of the conformal mapping $z=\ln w$. This function maps $S$ onto the rectangle $R\left\{\ln r \leqq x \leqq \ln (r+\Delta r),|y| \leqq \frac{1}{2} \Delta \theta\right\}$, the point $m=\sqrt{r(r+\Delta r)}$ of the $w$-plane being carried over into the center of $R$. The image of $w_{p, q}$ is given by

$$
z_{\tilde{p}, \bar{q}}=(\ln m)+\frac{1}{2} \not p h+\frac{1}{2} i \tilde{q} k \quad *
$$

where

$$
\begin{aligned}
& \tilde{p l}=2 \frac{\ln \left(1+\frac{p+1}{2} \frac{\Delta r}{r}\right)}{\ln \left(1+\frac{\Delta r}{r}\right)}-1 \\
& =p+\left(1-p^{2}\right) \frac{\Delta r}{4 r}\left\{1-\frac{1}{6}(3+2 p) \frac{\Delta r}{r}\right. \\
& \left.\quad+\frac{1}{24}\left(7+8 p+3 p^{2}\right)\left(\frac{\Delta r}{r}\right)^{2}+\cdots\right\},
\end{aligned}
$$

$$
\begin{aligned}
& \bar{q}=q, \\
& h=\ln \left(1+\frac{\Delta r}{r}\right), \quad k=\Delta \theta=\tau \Delta r .
\end{aligned}
$$

* For the sake of simplicity we use here the same notation as the one introduced in (1) which refers to the special case $m=1$. 
Now let

$$
\begin{aligned}
f(w)= & f\left(e^{2}\right)=F(z), \quad F\left(z_{\alpha, \beta}\right)=F_{\alpha, \beta}, \quad F_{\alpha, \beta}^{*}=\frac{1}{4}\left\{(1-\alpha)(1-\beta) F_{-1,-1}\right. \\
& \left.+(1+\alpha)(1-\beta) F_{1,-1}+(1-\alpha)(1+\beta) F_{-1,1}+(1+\alpha)(1+\beta) F_{1,1}\right\} .
\end{aligned}
$$

Then it follows from (3)

$$
F\left(z_{\tilde{p}, q}\right)-F_{\tilde{p}, \tilde{q}}^{*}=\frac{1}{8}\left(\tilde{p}^{2} h^{2}-\tilde{q}^{2} k^{2}-h^{2}+k^{2}\right) F^{\prime \prime}(\ln m)+\cdots
$$

or, in view of $(8)-(10)$,

$$
F\left(z_{\tilde{p}, \tilde{q}}\right)-F_{\tilde{p}, \tilde{q}}^{*}=\frac{1}{8}\left(\frac{\Delta r}{r}\right)^{2}\left(p^{2}-\tau^{2} r^{2} q^{2}-1+\tau^{2} r^{2}\right) F^{\prime \prime}(\ln m)+O\left[(\Delta r)^{3}\right] .
$$

Since furthermore

$$
\begin{aligned}
F_{\tilde{p}, \bar{q}}^{*}-F_{p, q}^{*} & =\frac{\tilde{p}-p}{4}\left\{-(1-q) F_{-1,-1}+(1-q) F_{1,-1}-(1+q) F_{-1,1}+(1+q) F_{1,1}\right\} \\
& =\frac{1-p^{2}}{8}\left(\frac{\Delta r}{r}\right)^{2} F^{\prime}(\ln m)+O\left[(\Delta r)^{3}\right]
\end{aligned}
$$

we have

$$
\begin{aligned}
& F\left(z_{\tilde{p}, \tilde{q}}\right)-F_{p, q}^{*}=\left(F_{\tilde{p}, \tilde{q}}^{*}-F_{p, q}^{*}\right)+\left(F\left(z_{\tilde{p}, \tilde{q}}\right)-F_{\tilde{p}, \tilde{q}}^{*}\right) \\
& \quad=\frac{1}{8}\left(\frac{\Delta r}{r}\right)^{2}\left\{\left(1-p^{2}\right) F^{\prime}(\ln m)+\left(p^{2}-\tau^{2} r^{2} q^{2}-1+\tau^{2} r^{2}\right) F^{\prime \prime}(\ln m)\right\}+O\left[(\Delta r)^{3}\right] .
\end{aligned}
$$

Thus, if we transform back to the $w$-plane, observing that

$F^{\prime}(\ln m)=m f^{\prime}(m), \quad F^{\prime \prime}(\ln m)=m f^{\prime}(m)+m^{2} f^{\prime \prime}(m), \quad m=r+O(\Delta r)$, we get

$$
\begin{aligned}
f\left(w_{p, q}\right) & -f_{p, q}^{*} \\
& =\frac{(\Delta r)^{2}}{8}\left\{\tau^{2}\left(1-q^{2}\right) r f^{\prime}(m)+\left(p^{2}-\tau^{2} r^{2} q^{2}-1+\tau^{2} r^{2}\right) f^{\prime \prime}(m)\right\}+O\left[(\Delta r)^{3}\right] .
\end{aligned}
$$

In order that the coefficient of $(\Delta r)^{2}$ be zero for all choices of $f(z)$ we must have $|p|=|q|=1$. Hence, in general, linear bivariate interpolation for all points of $S$ is affected with an error of $0\left[(\Delta r)^{2}\right]$ and there are no exceptional points other than the vertices of $S$ with higher order accuracy.

We note, however, that exceptional points will be present if we adopt a modified version of bivariate interpolation, namely, using $\tilde{p}$ as defined in (8) in place of $p$. It follows then from (11) that the interpolation error becomes of order $(\Delta r)^{3}$ for points $w_{p, q}$ along the curves

$$
p^{2}-\tau^{2} r^{2} q^{2}-1+\tau^{2} r^{2}=0 .
$$

Moreover, if $\Delta \theta=\ln (1+(\Delta r / r))$, that is, if the conformal image $R$ of $S$ is a square, this modification leads to an error of order $(\Delta r)^{4}$ at the point 
$m=\sqrt{r(r+\Delta r)}$ of $S$. These facts may occasionally be used to improve the accuracy of bivariate polar interpolation.

American University

Washington, District of Columbia

1. V. N. Faddeeva \& N. M. Terent'ev, Tablicy značenii funkcii

$w(z)=e^{-z^{2}}\left(1+\frac{2 i}{\sqrt{ } \pi} \int_{0}^{z} e^{t^{2}} d t\right)$ ot kompleksnogo argumenta, Gosudarstv. Izdat. Tehn.-Teor.

Lit., Moscow, 1954.

2. Harvard University, Tables of the function arcsinz, The annals of the Computation Laboratory, v. 40, 1956.

3. K. A. KARPov, Tablicy funkcii $w(z)=e^{-z^{2}} \int_{0}^{z} e^{x^{2}} d x v$ kompleksnoi oblasti, Izdat. Akad. Nauk SSSR, Moscow, 1954.

4. K. A. Karpov, Tablicy funkcii $\mathrm{F}(z)=\int_{0}^{z} e^{x^{2}} d x v$ kompleksnoi oblasti, Izdat. Akad. Nauk SSSR, Moscow, 1958.

5. National Bureau of Standards, Table of the Bessel Functions $J_{0}(z)$ and $J_{1}(z)$ for complex arguments, 2nd ed., Columbia University Press, New York, 1947.

6. National Bureau of Standards, Table of the Bessel Functions $Y_{0}(z)$ and $Y_{1}(z)$ for complex arguments, Columbia University Press, New York, 1950.

7. National Bureau of Standards, Table of the gamma function for complex arguments, Applied Math. Series 34, 1954.

8. National Bureau of Standards, Tables of the exponential integral for complex arguments, Applied Math. Series 51, 1958.

9. H. E. SALzer, "Coefficients for polar complex interpolation," J. Math. Phys., v. 29, 1950 , p. $96-104$.

10. H. E. SALzER, "Osculatory interpolation in the complex plane," J. Research, NBS, v. 54, Report 2587,1955 , p. $263-266$.

11. H. UnGER, "Lagrange-Hermitesche Interpolation im Komplexen," Z. angew. Math. Phys., v. 3, 1952, p. 51-65.

12. Added in proof. In a private communication E. Kreyszig and J. Todd have studied the case of bivariate quadratic interpolation with respect to nine pivotal points $z_{p, q}=p h+$ $i q k(k=\kappa h$ and $p, q$ running independently through $-1,0,1)$. For the interpolation error they obtain the expression

$$
\begin{aligned}
f\left(z_{p, q}\right)-f_{p, q}^{*}=\left[p\left(p^{2}-1\right)-i \kappa^{3} q\right. & \left.\left(q^{2}-1\right)\right] \frac{h^{3}}{6} f_{0}^{\prime \prime \prime}+\left[p^{2}\left(p^{2}-1\right)\right. \\
& \left.+\kappa^{4} q^{2}\left(q^{2}-1\right)+4 i \kappa p q\left\{p^{2}-1-\kappa^{2}\left(q^{2}-1\right)\right\}\right] \frac{h^{4}}{24} f_{0}^{\mathrm{iv}}+o\left(h^{4}\right),
\end{aligned}
$$

from which they observe that no exceptional points exist, other than the pivitol points, where the error is of order $h^{4}$. 